ARTICLE

Received 26 Feb 2015 | Accepted 20 May 2015 | Published 6 Jul 2015

DOI: $10.1038 /$ ncomms 8551

OPEN

\title{
The GATA transcription factor GtaC regulates early developmental gene expression dynamics in Dictyostelium
}

\author{
Balaji Santhanam ${ }^{1,2, \star}$, Huaqing $\mathrm{Cai}^{3}$, Peter N. Devreotes ${ }^{3}$, Gad Shaulsky ${ }^{2} \&$ Mariko Katoh-Kurasawa ${ }^{2, \star}$
}

In many systems, including the social amoeba Dictyostelium discoideum, development is often marked by dynamic morphological and transcriptional changes orchestrated by key transcription factors. However, efforts to examine sequential genome-wide changes of gene regulation in developmental processes have been fairly limited. Here we report the developmental regulatory dynamics of GtaC, a GATA-type zinc-finger transcription factor, through the analyses of serial ChIP- and RNA-sequencing data. GtaC is essential for developmental progression, decoding extracellular cAMP pulses during early development and may play a role in mediating cell-type differentiation at later stages. We find that GtaC exhibits temporally distinctive DNA-binding patterns concordant with each developmental stage. We identify direct GtaC targets and observe cotemporaneous GtaC-binding and developmental expression regulation. Our results suggest that GtaC regulates multiple physiological processes as Dictyostelium transitions from a group of unicellular amoebae to an integrated multicellular organism.

\footnotetext{
${ }^{1}$ Graduate Program in Structural and Computational Biology and Molecular Biophysics, Baylor College of Medicine, Houston, Texas 77030, USA.

${ }^{2}$ Department of Molecular and Human Genetics, Baylor College of Medicine, Houston, Texas 77030, USA. ${ }^{3}$ Department of Cell Biology, School of Medicine, Johns Hopkins University, Baltimore, Maryland 21205, USA. * These authors contributed equally to this work. Correspondence and requests for materials should be addressed to G.S. (email: gadi@bcm.edu) or to M.K.-K. (email: mkatoh@bcm.edu).
} 
$\mathrm{D}$ evelopment involves the sequential execution of molecular events achieved by gene-regulatory networks ${ }^{1,2}$ that underlie precise changes in spatiotemporal gene expression patterns $s^{3-5}$. Gene-regulatory networks are important biological control systems in which several input and feedback signals are processed to render optimal cellular responses, often by altering overall cellular physiology ${ }^{1}$. Gene expression regulation is mediated through specific interactions between transcription factors (TFs) and cis-regulatory DNA elements. Chromatin immunoprecipitation followed by high-throughput sequencing (ChIP-seq) is widely used to assay in vivo TF-DNA interactions. This approach provides a genome-wide view of TF-binding preferences, making the discovery of functional cis-regulatory DNA elements possible ${ }^{6}$. Genetic perturbation of TFs followed by quantitative measurements of global transcriptional profiles offer another dimension in understanding gene regulation by TFs. Analysing binding preferences of TFs, transcriptome profiling coupled with specific genetic perturbations and finally integrating these binding and transcriptome data aid in providing a comprehensive view of functional regulatory relationships between TFs and their direct targets ${ }^{7}$.

Transcriptional changes accompanying Dictyostelium development have been well documented ${ }^{8}$ and efforts have been made to ascertain the developmental roles of several $\mathrm{TFs}^{9}$, including GbfA (G-box binding factor A) ${ }^{10}$, STATa (signal transducers and activators of transcription a) ${ }^{11}$, SrfA (MADS-box containing serum response factor $\mathrm{A})^{12}$ and CudA (culmination defective $\overline{\mathrm{A}})^{13}$. Upon starvātion, unicellular $D$. discoideum amoebae initiate a developmental programme and organize into multicellular structures by aggregating in response to $3^{\prime}-5^{\prime}$-cyclic adenosine monophosphate (cAMP) waves that propagate through the field of cells. After aggregation, cell-type differentiation occurs accompanied by dynamic morphological changes and the developmental process ends in the formation of fruiting bodies consisting of two major cell types-spores and stalks ${ }^{14,15}$. D. purpureum is an evolutionarily distant dictyostelid species whose developmental morphology is strikingly similar to that of D. discoideum. Both species use cAMP as a chemoattractant and their developmental transcriptomes are remarkably conserved ${ }^{8,16,17}$. One of the conserved transcripts is of $g t a C$, which is abundant in both $D$. discoideum and $D$. purpureum during cAMP-mediated aggregation and encodes a GATA-type $\mathrm{TF}^{8}$.

$\mathrm{GtaC}$ is a key regulator that decodes extracellular cAMP pulses and establishes downstream signalling pathways, ensuring timely development. During early development, GtaC shuttles between the nucleus and cytoplasm in response to, and out-of-phase with, extracellular cAMP pulses. Cells lacking gtaC are defective in aggregation and in morphological and transcriptional progression. Ectopic expression of $\mathrm{GtaC}$ fused with green fluorescent protein (GtaC-GFP) in the gtaC-null (knockout, KO) strain complements these defects. The expression of a mutated $\mathrm{GtaC}$, in which all four cysteine residues of the zinc-finger domain were substituted with serine residues, does not complement the developmental defects of the $\mathrm{KO} \operatorname{strain}^{18}$. GtaC may also play a role in cell differentiation during later stages of development ${ }^{19}$. Curiously, gtaC transcript abundance increases twice during development. Once during early aggregation and once starting at late aggregation and continuing through the end of development (dictyExpress (http://dictyexpress.biolab.si/)). This observation is broadly consistent with the early and late phenotypes of the gtaC-mutant strains ${ }^{18,19}$. The possible dependence between the early and late phenotypic manifestations of $g t a C$ has not been examined yet.

Dictyostelium development is accompanied by sweeping changes in gene expression ${ }^{8,9}$, but the regulatory events that mediate these changes are not well understood ${ }^{20,21}$. Defects in gtaC manifest as an early developmental phenotype ${ }^{18}$, making it an ideal candidate to begin elucidating the Dictyostelium developmental gene-regulatory network. In this study, we analyse sequential genomic occupancy data of the wild-type (WT) and cysteine-substituted (CS) forms of GtaC using ChIPseq to explore the developmental roles of GtaC further. At contemporaneous developmental stages, we also analyse serial transcriptional profiles using RNA sequencing (RNA-seq). By integrating these binding and transcriptome data, we identify $\mathrm{GtaC}$ target genes. We find that $\mathrm{GtaC}$ targets include developmentally essential TFs and genes involved in cAMP signalling during early development and those relating to celltype specification at later stages. Our results suggest that $\mathrm{GtaC}$ regulates early development by controlling different physiological processes concordant with developmental progression in Dictyostelium.

\section{Results}

Temporal regulation of GtaC-DNA interactions. GtaC is essential for early development in $D$. discoideum and its nuclear localization is regulated by pulsatile cAMP signals during aggregation ${ }^{18}$. To examine the association of $\mathrm{GtaC}$ with genomic DNA, we performed ChIP-seq using the CM strain, in which ectopic expression of $\mathrm{GtaC}$-GFP complements the $\mathrm{gtaC}$-null mutation, and used the GFP-expressing $\mathrm{KO}$ strain as a negative control to exclude potential binding by GFP (Supplementary Fig. 1a, Supplementary Table 1). We developed the cells and collected samples during initial starvation (S), early aggregation (E) - as waves of cAMP are observed - and late aggregation (L) as the cAMP concentration increases. We identified 2,091 genes whose promoter regions were bound by $\mathrm{GtaC}$ (greater than fourfold enrichment in coverage between sample and control ChIP-seq experiments) at any of the three time points (Supplementary Fig. 1b), more than $70 \%$ of which were bound at early aggregation (clusters ii, iii and iv in Fig. 1a, Supplementary Table 2). A representative example is shown in Fig. 1b, wherein the upstream regions of the cupF and DDB_G0289831 loci were associated with GtaC during starvation-sensing and early aggregation, but not during late aggregation. We found 462 genes, including $d c s A$ (Fig. 1c), which were bound by $\mathrm{GtaC}$ only during late aggregation (cluster $\mathrm{v}$ in Fig. 1a). In fact, more than $60 \%$ of the genes were bound only at one stage (clusters i, iii or v in Fig. 1a, Supplementary Table 2), indicating that $\mathrm{GtaC}$ exhibits highly dynamic DNA binding throughout early development. Two central components of the cAMP signalling apparatus - the early adenylyl cyclase gene acaA and the early cAMP-receptor gene carA, both of which utilize alternative promoters ${ }^{22,23}$, were bound by $\mathrm{GtaC}$ at all three stages (cluster iv in Fig. 1a). The temporal GtaC-binding patterns were consistent with both the promoter usage and developmental regulation previously observed in these genes (Supplementary Fig. 2) 22,23 . Overall, these results support the hypothesis that GtaC mediates cAMP signalling in this system ${ }^{18}$ by regulating the expression of cAMP-related genes.

GtaC-DNA binding. GtaC is a member of the GATA TF family. It contains a $\mathrm{Cys}_{2}-\mathrm{Cys}_{2}$-type IV zinc-finger domain that mediates DNA binding with a zinc ion ${ }^{24,25}$. The CS strain carries a mutated form of $\mathrm{GtaC}$ in which all four cysteine residues (C4) of the zincfinger domain were substituted with serine residues $\left(\mathrm{GtaC}^{\mathrm{C}-\mathrm{S}}\right)$. Expression of $\mathrm{GtaC}^{\mathrm{C}-\mathrm{S}}-\mathrm{GFP}$ in the KO strain does not complement the defects in aggregation and morphological progression (Supplementary Table 1$)^{18}$. We expected that this mutant form of $\mathrm{GtaC}$ would lack DNA binding. To test this 
a

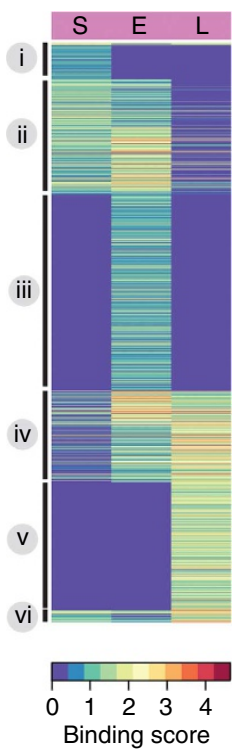

b

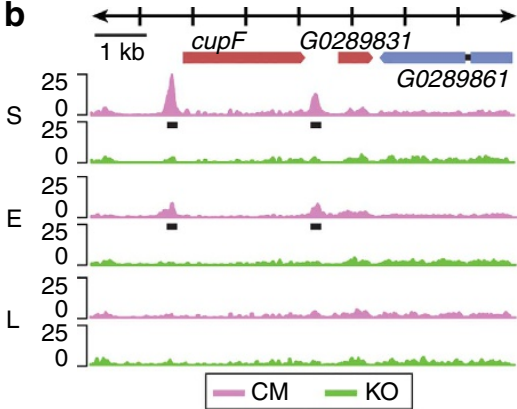

c

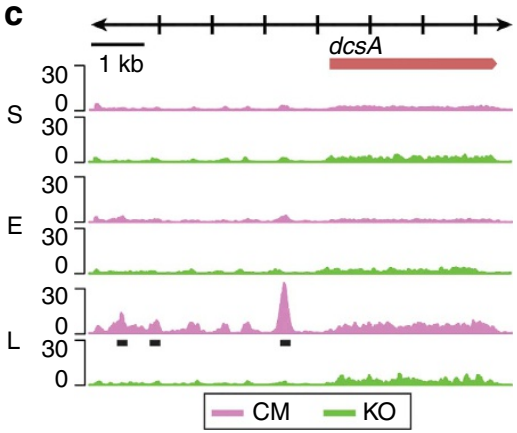

Figure 1 | Genome-wide occupancy of GtaC during development.

(a) Heatmap representation of binding scores ( $\log _{2}$-transformed, scale indicated) of genes bound by $\mathrm{GtaC}$ in their promoter regions during three developmental stages (S-starvation-sensing, E-early aggregation and $L$-late aggregation; $n=2,091$ ). Each row represents a gene and each column represents a developmental stage. Rows are ordered on the basis of hierarchical clustering with optimized leaf ordering and grouped (black lines, left) on the basis of temporal patterns of binding events. $(\mathbf{b}, \mathbf{c})$ Representative examples of ChIP-seq patterns (scaled coverage on the $y$ axis, DNA length in kilobases on the $x$ axis, as indicated at the top). The colours of the tracks indicate the genotypes of the cells (purple: gtaC-null complemented with ectopic GtaC-GFP (CM), green: gtaC-null GFP $(\mathrm{KO})$ ). All the predicted gene models within the genomic region are shown. The exons are coloured depending on the orientation of the gene (red: positive, blue: negative) and introns are shown as black lines. Binding data are shown during starvation-sensing $(\mathrm{S})$, early aggregation $(E)$ and late aggregation around the (B) CupF and DDB_G0289831 loci on chromosome 5, and the $(C)$ dcsA locus on chromosome 1. Identified peaks are indicated (black lines).

hypothesis, we performed ChIP-seq in the CS strain and found that most of the genes bound by GtaC showed no detectable $\mathrm{GtaC}^{\mathrm{C}-\mathrm{S}}$ binding. For example, the upstream regions of the cAMP-receptor gene $\operatorname{carC}$ were occupied by $\mathrm{GtaC}$, but not by $\mathrm{GtaC}^{\mathrm{C}-\mathrm{S}}$, at late aggregation (Fig. 2a). Surprisingly, we identified 678 genes that showed $\mathrm{GtaC}^{\mathrm{C}-\mathrm{S}}$ binding in their promoter regions (Supplementary Table 2, Supplementary Fig. 1c), most of which $(84 \%)$ were also bound by $\mathrm{GtaC}$. These results suggest that the zinc-finger $\mathrm{C} 4$ mutation changed the propensity but did not completely abolish the ability of $\mathrm{GtaC}$ to bind DNA.

To assess the specificity of these interactions, we searched for positional and temporal concordance between $\mathrm{GtaC}^{\mathrm{C}-\mathrm{S}}$ - and GtaC-binding events. The binding positions of $\mathrm{GtaC}^{\mathrm{C}-\mathrm{S}}$ and GtaC were remarkably similar in most promoter regions (Supplementary Fig. 3a; top panel), suggesting that $\mathrm{GtaC}^{\mathrm{C}-\mathrm{S}}$ DNA binding represents a mode of normal GtaC-DNA interactions that are independent of the $\mathrm{C} 4$ residues in the zinc-finger domain. The extent of overlap between $\mathrm{GtaC}^{\mathrm{C}-\mathrm{S}}$ - and GtaC-DNA binding was high during early aggregation (83\%) but lower during late aggregation (36\%; Supplementary Table 2). In fact, most of the genes bound by both $\mathrm{GtaC}^{\mathrm{C}-\mathrm{S}}$ and $\mathrm{GtaC}$ at the
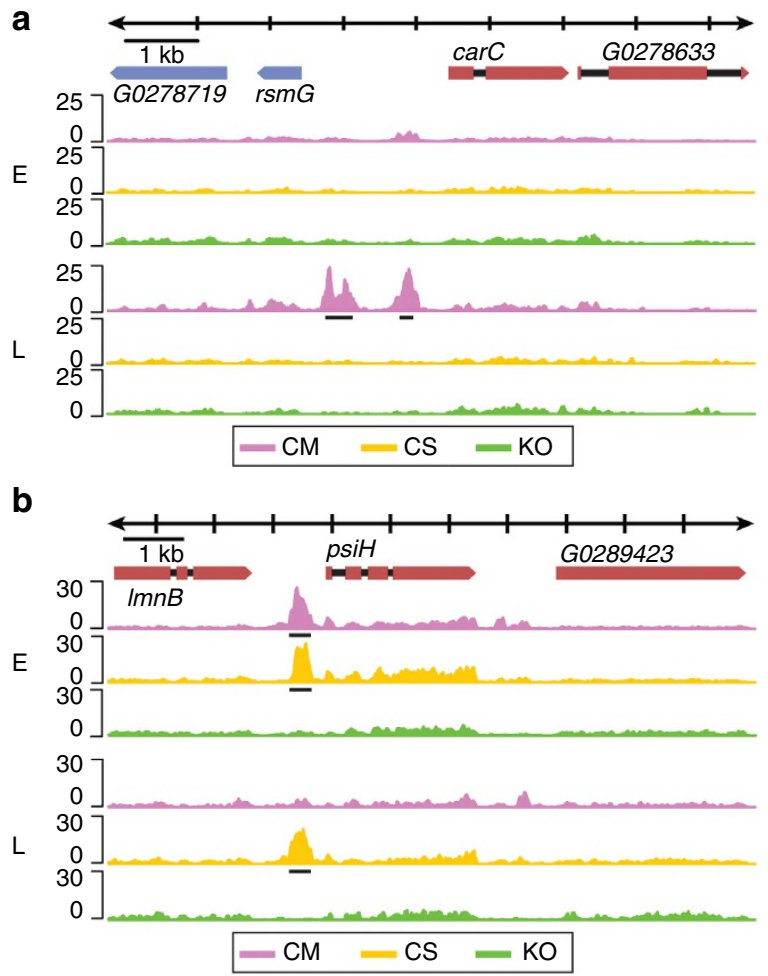

C

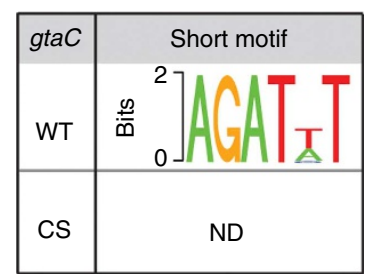

Figure 2 | The zinc-finger cysteine residues (C4) affect the DNA-binding specificity of GtaC. Representative examples of ChIP-seq patterns (scaled coverage on the $y$ axis, DNA length in kilobases on the $x$ axis, as indicated at the top). The colours of the tracks indicate the genotypes of the cells (purple: gtaC-null complemented with ectopic GtaC-GFP (CM), yellow: gtaC-null ectopically expressing the GtaC ${ }^{\mathrm{C}-\mathrm{S}}$-GFP (CS), green: gtaC-null GFP $(\mathrm{KO})$ ). All the predicted gene models within the genomic region are shown. The exons are coloured depending on the orientation of the gene (red: positive, blue: negative) and introns are shown as black lines. Binding data are shown during early aggregation (E) and late aggregation $(L)$ around the (a) carC locus on chromosome 3 and (b) psiH locus on chromosome 5. Identified peaks are indicated (black lines). (c) We identified enriched motifs using MEME-ChIP56 on the 600 most enriched peaks of the WT (top row) and the CS (bottom row) gtaC alleles. Information content (bits) is shown on the $y$ axis. Motifs identified through DREME (short motif) are shown ${ }^{57,58}$. ND indicates that no motifs were detected.

earlier time point were bound by only $\mathrm{GtaC}^{\mathrm{C}-\mathrm{S}}$ at the later time point (Supplementary Fig. 3a; bottom panel). A representative example is shown in Fig. $2 b$, where the upstream region of the $p s i H$ locus exhibited GtaC-binding at early aggregation but not at late aggregation, whereas $\mathrm{GtaC}^{\mathrm{C}-\mathrm{S}}$ was bound at both times. These results are consistent with the lack of further developmental progression observed in the CS strain and also suggest that $\mathrm{GtaC}^{\mathrm{C}-\mathrm{S}}$ might misregulate its target genes.

To investigate GtaC-binding specificity, we searched for over-represented, short DNA sequences in genomic regions associated with GtaC- or $\mathrm{GtaC}^{\mathrm{C}-\mathrm{S}}$ peaks. We found enrichment for a GATA-like motif only in regions bound by the WT GtaC (Fig. 2c, Supplementary Fig. 3b). 
GtaC regulates developmental gene expression. To test whether GtaC-binding events were accompanied by gene expression changes, we used our published RNA-seq data of WT, CM and $\mathrm{KO}$ strains ${ }^{18}$ and added a few data sets. We first obtained the developmental transcriptome of the CS strain. Since we observed $\mathrm{GtaC}$ binding at the late aggregation stage, we also obtained the transcriptomes of all the strains at this stage (Supplementary Data 1, Supplementary Fig. 4). To visualize the differences among the transcriptomes, we used multidimensional scaling (MDS), which provides a low dimensional projection ${ }^{26}$ that best represents the relative distances between individual transcriptomes in their original higher dimensional space (Fig. 3a). We observed that the $\mathrm{CS}$ and KO transcriptomes clustered close together. Interestingly, all the CS and KO developmental transcriptomes, other than at $0 \mathrm{~h}$, clustered together with the 1- and 2-h transcriptomes of WT and CM strains at the starvation-sensing stage, indicating a lack of further transcriptional progression. These observations are consistent with the developmental arrest observed in both the $\mathrm{KO}$ and CS strains ${ }^{18}$ before the aggregation stage, which normally corresponds to the onset of cAMP signalling. They also indicate that, despite the presence of $\mathrm{GtaC}^{\mathrm{C}-\mathrm{S}}$ in the cells and its ability to bind DNA, the global developmental transcriptomes of the CS strain showed little difference from that of the KO. The WT and $\mathrm{CM}$ transcriptomes were similar to one another at each of the time points. They exhibited clear temporal progression such that their transcriptomes at starvation-sensing ( 1 and $2 \mathrm{~h}$ ) were closer to each other than to the transcriptomes at early aggregation $(5 \mathrm{~h})$ or late aggregation $(8 \mathrm{~h})$ stages. Overall, these results suggest that the developmental consequences of carrying various forms of $g t a C$ are accompanied and likely caused by overt transcriptional differences between the strains that express them.

We chose to focus on genes that exhibited developmentally regulated changes in their mRNA abundance within each strain over time and between strains at any time point. We found that a majority $(\sim 70 \%)$ of the genes in KO and CS strains did not show any significant developmental changes in mRNA abundance, consistent with the lack of transcriptional and morphological progression in these strains (Fig. $3 \mathrm{~b})^{18}$. It is therefore likely that most $\mathrm{GtaC}^{\mathrm{C}-\mathrm{S}}-\mathrm{DNA}$-binding events have no direct role in the regulation of gene expression. We identified 1,188 genes that were differentially expressed between strains with normal (WT, CM) and aberrant (KO, CS) developmental progression and also developmentally regulated in both WT and CM strains. Using hierarchical clustering, we identified five broad clusters of genes that exhibited coordinated changes in their mRNA abundance over time (Fig. 3b). Genes in clusters i, ii and iii, appeared to be turned on at vegetative growth $(\mathrm{V})$, starvation-sensing $(\mathrm{S})$ or early aggregation (E) stages, respectively, but were turned off by the late aggregation (L) stage in both WT and CM strains (WT and CM in Fig. 3b). This downregulation was compromised in both $\mathrm{KO}$ and CS strains (KO and CS in Fig. 3b). Genes in clusters iv and $\mathrm{v}$ were upregulated at early (E) and late aggregation (L) stages, respectively, in both WT and CM strains, but not in the KO or CS strains. Some of the transcriptional differences between the WT (or CM) and the KO (or CS) strains, especially at late aggregation, may represent cumulative effects due to the early developmental defect observed in both the $\mathrm{KO}$ and CS strains. Notwithstanding, these results suggest that $\mathrm{GtaC}$ is a key factor that affects different groups of developmentally regulated genes, influencing both timing and the up/downregulation of their expression.

Gene-regulatory consequences of $\mathrm{GtaC}$ and $\mathrm{GtaC}^{\mathrm{C}-\mathrm{S}}$ binding. To explore the regulatory effects of $\mathrm{GtaC}$ and $\mathrm{GtaC}^{\mathrm{C}-\mathrm{S}}$ binding, we tested the relationship between binding events on the basis of the ChIP-seq data and transcriptional changes in the RNA-seq
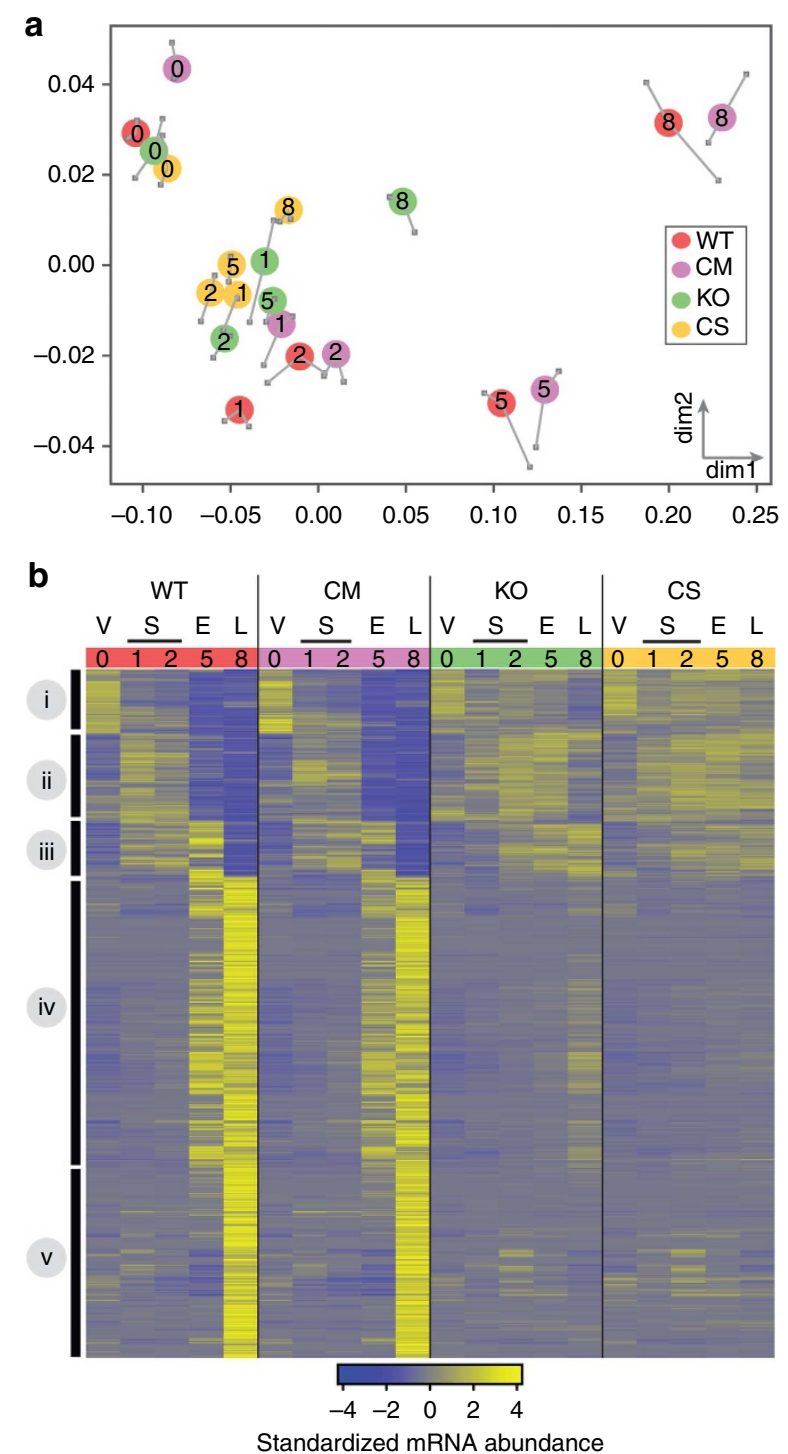

Figure 3 | Developmental regulation of GtaC-dependent transcription.

(a) MDS visualization of distances between transcriptomes. Each coloured circle represents the average transcriptome of two biological replicates (grey squares and whiskers) and numbers inside the circles represent the developmental time (h). Distances on the two-dimensional plane are inversely proportional to the similarity between the transcriptomes. Strains: WT-wild type, CM-gtaC-null complemented with ectopic GtaC-GFP, KO-gtaC-null, CS-gtaC-null ectopically expressing the GtaC C-S-GFP. (b) Heatmap representation of standardized mRNA abundance of genes $(n=1,188$; scale indicated) that show significant changes between strains that exhibit normal (WT and CM) and aberrant (KO and CS) development. Each row represents a gene and each column represents a transcriptome. Rows are grouped by hierarchical clustering and broad clusters are shown as solid black lines (left). The columns are grouped by strain (coloured panels on top, red: $\mathrm{WT}$, purple: $\mathrm{CM}$, green: $\mathrm{KO}$, yellow: $\mathrm{CS}$ ) and ordered by time ( $h$, indicated above each column). The time line corresponds to the developmental stage in the WT strain as indicated (V: vegetative growth, $\mathrm{S}$ : starvation-sensing, E: early aggregation and L: late aggregation).

data at the corresponding time points. We identified genes bound by $\mathrm{GtaC}$ and also differentially expressed between CM and $\mathrm{KO}$ strains at the two aggregation stages (E: 952 genes, Fig. 4a; L: 621 genes, Fig. 4b). In addition, we identified genes bound by $\mathrm{GtaC}^{\mathrm{C}-\mathrm{S}}$ and differentially expressed between the CS and KO strains at these two time points (E: 38 genes, Fig. 4a; L: 231 genes, Fig. 4b). 
a

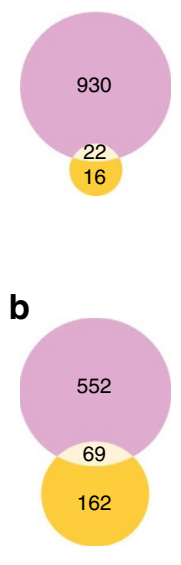

C

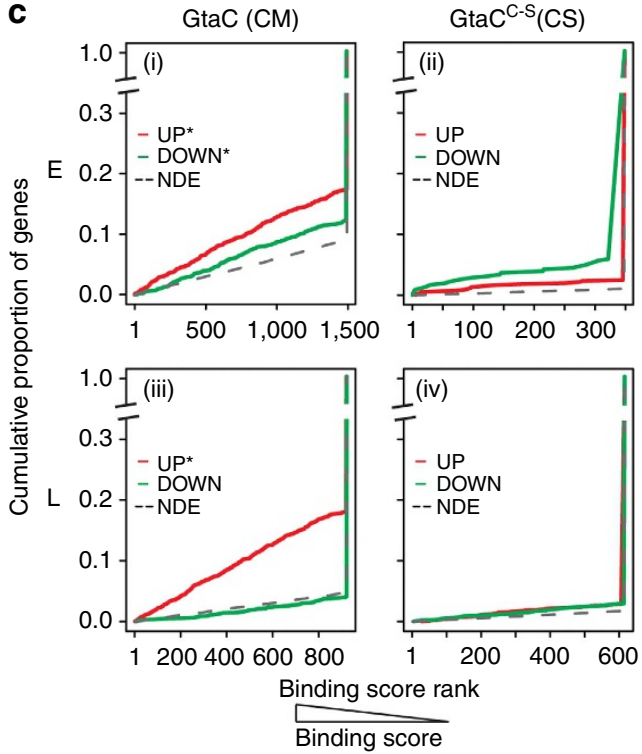

d

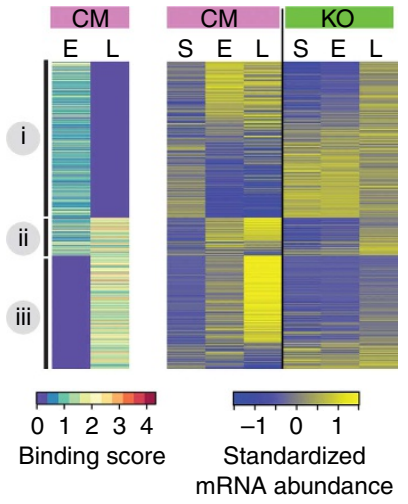

Figure 4 | Regulatory consequences of $\mathbf{G t a C}$ and $\mathbf{G t a C}^{\mathbf{C}-\mathbf{S}}$ promoter-binding events on gene regulation. (a,b) Venn diagrams showing the overlap between genes bound by $\mathrm{GtaC}$ and differentially expressed between gtaC-null cells complemented with ectopic GtaC-GFP (CM) and gtaC-null (KO) cells (purple circle) and, genes bound by $\mathrm{GtaC}^{\mathrm{C}-\mathrm{S}}$ and differentially expressed between gtaC-null cells ectopically expressing $\mathrm{GtaC} \mathrm{C}^{\mathrm{C}-\mathrm{S}}$-GFP (CS) and $\mathrm{KO}$ cells (yellow circle) during early (a) and late (b) aggregation. (c) Plots showing the cumulative proportions of genes ( $y$ axis) at a given binding score (ranked; $x$ axis) of $\mathrm{GtaC}$ or $\mathrm{GtaC}^{\mathrm{C}-\mathrm{S}}$ at early aggregation (E; (i), (ii)) and late aggregation ( $\mathrm{L}$; (iii), (iv)). All genes are categorized as upregulated (UP, red lines), downregulated (DOWN, green lines) or NDE (black dashed lines) in CM (first column) or CS (second column) compared with KO cells. The UP and DOWN cumulative distributions are compared with the corresponding NDE distribution using the Kolmogorov-Smirnov test $(P$ values $<0.001$ indicated by * in (i) and (iii)). (d) The heatmaps represent GtaC-binding scores (log 2 -transformed; scale indicated, left panel) and standardized mRNA abundance (scale indicated, right panel) of genes bound only by $\mathrm{GtaC}$ at early aggregation ( $\mathrm{E}$; clusters i-ii) or late aggregation ( $\mathrm{L}$; clusters ii-iii). Each row represents a gene and genes were grouped by their binding patterns. Each column represents a strain (coloured panels on top, purple: $\mathrm{CM}$, green: $\mathrm{KO}$ ) and time point (S: starvation-sensing, E: early aggregation and L: late aggregation).

We found that a small but significant fraction of these two gene sets overlapped at either time points (22 in Fig. $4 \mathrm{a}$ and 69 in Fig. 4b).

To assess these data more quantitatively, we tested whether binding events in the promoters were more likely associated with up- or downregulation of gene expression ${ }^{7}$. We first categorized all genes as upregulated (UP), downregulated (DOWN) or not differentially expressed (NDE) in CM or CS strains compared with the KO strain. We then compared binding score distributions of genes categorized UP or DOWN with those categorized NDE, in each strain (Fig. 4c, Supplementary Table 3). We found that GtaC-binding events were significantly associated with both up- and downregulation of gene expression at early aggregation (Fig. $4 \mathrm{c}(\mathrm{i}))$ and only with upregulated genes at the late stage (Fig. $4 \mathrm{c}(\mathrm{iii})$ ). In contrast, $\mathrm{GtaC}^{\mathrm{C}-\mathrm{S}}$ binding was not associated with up- or downregulation of gene expression at both stages (Fig. 4c(ii,iv)). In fact, of the 245 genes (from Fig. 4a,b) that were bound and differentially expressed in CS compared with KO at either time point, only two genes displayed similar expression levels between CM and CS strains. Taken together, these results suggest that, despite the apparent interaction of $\mathrm{GtaC}^{\mathrm{C}-\mathrm{S}}$ with DNA, the binding events mostly result in faulty regulation of target gene expression.

To further evaluate the regulatory consequences of $\mathrm{GtaC}$ binding to GATA-like sequences, we chose genes bound only by $\mathrm{GtaC}$ at early or late aggregation and tested whether GtaC binding to the motif was associated with coordinated transcriptional changes in $\mathrm{CM}$ but not in $\mathrm{KO}$ strains. About half the genes bound by $\mathrm{GtaC}$ only at early aggregation showed increased abundance in $\mathrm{CM}$ at this time compared with starvation-sensing, and this upregulation was not observed in $\mathrm{KO}$ (Fig. $4 \mathrm{~d}$, cluster i). The other half, however, was low at early aggregation in CM and remained low until the late stage (Fig. 4d, cluster i). This observation suggests that the low abundance at late aggregation is likely due to indirect effects. On the other hand, most genes bound by $\mathrm{GtaC}$ at late aggregation were clearly upregulated at this time in the CM, but not in the KO (Fig. 4d, clusters ii-iii). These results suggest that $\mathrm{GtaC}$ binding to GATA-like sequences has a direct role in upregulating gene expression (Fig. 4c(i,iii), Fig. 4d); however, other mechanisms may be required to differentially regulate target genes at different stages.

Identifying direct targets of GtaC. We defined direct targets of $\mathrm{GtaC}$ as genes that were associated with $\mathrm{GtaC}$ binding and showed differential mRNA patterns between the $\mathrm{CM}$ and $\mathrm{KO}$ strains. We used a rank-product (RP) score to integrate these two parameters ${ }^{7}$ such that genes ranked high in both the binding and transcriptome data sets had smaller GtaC-RP scores. We considered the 561 genes with $\mathrm{GtaC}-\mathrm{RP}<0.005$ at least in one of the three time points as likely direct targets of $\mathrm{GtaC}$ ( $\mathrm{GtaC}$ targets; Supplementary Data 2). We also tested whether differences in transcript abundance of GtaC targets between CM and WT could be the result of ectopic expression of GtaC-GFP in CM. Comparing the two strains revealed that overall, transcript abundance genome wide, including those of $\mathrm{GtaC}$ targets, were remarkably consistent at each of the time points tested (Fig. 3a, Supplementary Fig. 5 (Spearman's correlation $(\mathrm{SC})>0.95)$ ). These findings suggest that GtaC-dependent transcriptional regulation in both the $\mathrm{CM}$ and WT strains are quite similar and therefore the GtaC-GFP protein is a good representative of the native $\mathrm{GtaC}$ function. As a measure of functional correlation between temporal patterns of $\mathrm{GtaC}$ binding and gene expression, we calculated their correlation for each gene. More than $60 \%$ of 
the GtaC targets showed similar trends in both (absolute ranked correlation $>0.5$ ), indicating that $\mathrm{GtaC}$ binding and expression of downstream genes were cotemporaneous and suggesting a causative relationship between $\mathrm{GtaC}$ binding and regulated gene expression.

To explore the biological processes regulated by GtaC, we examined gene annotations within the putative direct targets. In a direct comparison with previously defined gene sets, we found the sets of 'cAMP-responsive'27 and 'cell-type-enriched" 8 genes to be significantly over-represented among GtaC targets (Supplementary Table 4). These observations are broadly consistent with previous descriptions of the two major functions of $\mathrm{GtaC}$ during development: cAMP-mediated signalling $^{18}$ and cell-type specification ${ }^{19}$. Our results further suggest that at least some of these genes are directly regulated by GtaC. The set of genes with known developmental functions (obtained from dictyBase (http://dictybase.org/)) and the set of putative $\mathrm{TFs}^{9}$ were also over-represented (Supplementary Table 4), suggesting that the regulatory modules involving GtaC and its direct targets play critical roles in development.

D. discoideum and D. purpureum are two evolutionarily distant dictyostelid species whose aggregation is mediated by cAMP and, their gtaC transcript levels are abundant during the aggregative phases of development ${ }^{8,16,17}$. Hence, we tested for the enrichment of $D$. purpureum orthologues among GtaC-dependent target genes in $D$. discoideum. Genes bound by GtaC during the aggregative stages were significantly enriched in the set of orthologous genes. Furthermore, a significant proportion of GtaC targets in the set of D. purpureum orthologues were coordinately expressed during development across the two species ${ }^{8}$. Given their strikingly similar developmental morphologies and transcriptomes, despite the evolutionary distance between the two species, our results suggest evolutionarily conserved developmental roles for GtaC-dependent regulatory modules in these dictyostelids.

To broaden the association between GtaC targets and Dictyostelium developmental processes, we performed an unbiased gene ontology (GO) enrichment analysis and identified enriched GO terms at each time point. The identified GO terms were largely associated with the key developmental processes'aggregation' at early aggregation and 'cell differentiation' at late aggregation, for example, as shown in Fig. 5, Supplementary Data 3. We also discovered annotations related to calcium ion binding and homeostasis, signalling through G-protein-coupled

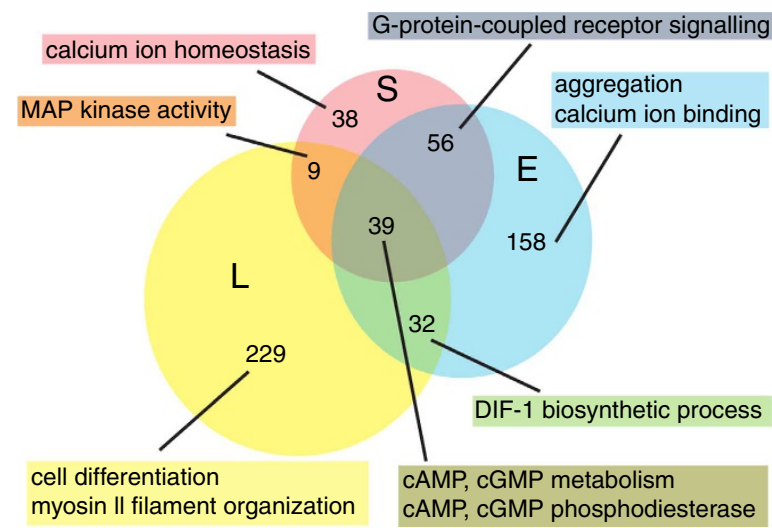

Figure 5 | Identifying direct targets of GtaC. A Venn diagram showing the extent of overlap between $\mathrm{GtaC}$ target genes $(n=561)$ at the three developmental times ( $\mathrm{S}$-starvation-sensing, red; E-early aggregation, blue; $L$-late aggregation, yellow). The number of overlapping genes and the prominent GO terms ( $P$ values $<0.01$; Fisher's exact test) enriched in each subset are indicated. receptors and mitogen activated protein (MAP) kinases as well as cyclic nucleotide metabolism and degradation and differentiation inducing factor 1 (DIF-1) biosynthesis. These results provide novel insights into the role of GtaC in development and shed light on critical GtaC-controlled components underlying the generegulatory networks governing multiple physiological processes in Dictyostelium development.

\section{Discussion}

In this paper, we showed that GtaC exhibits distinct temporal binding patterns during early development, thus alluding to multiple developmental roles. Such variable binding specificities have been reported in other systems, where they appear to result from various regulatory effectors ${ }^{28,29}$. Multicellular development is marked by complex signalling events that alter the physiological states of cells concomitantly with morphological progression $^{30,31}$, and these cellular stimuli often act to alter the localization and activity of $\mathrm{TFs}^{32}$. Temporally regulated binding events are likely regulated by the combined action of many TFs coupled with changes to the chromatin architecture, ultimately resulting in gene expression changes ${ }^{2}$. In the case of GtaC, its nuclear localization and hence transcriptional activity during early development are dependent on $\mathrm{CAMP}^{18}$; however, it is unclear what protein modifications and/or epigenetic effects are responsible for the observed changes in binding patterns.

Here we found that the zinc-finger domain of $\mathrm{GtaC}$ was essential for specific binding to the GATA motif. Recent proteinbinding microarray experiments revealed that the GtaC DNAbinding domain showed higher-affinity binding to a consensus 'GATC' sequence, than to 'GATA' sequences ${ }^{33}$. It should be noted that the strengths of TF binding cannot be inferred from ChIP-seq experiments and the differences in the identified motifs could also be attributed to using the DNA-binding domain in the in vitro protein-binding microarray experiments ${ }^{33}$ compared with the full-length GtaC-GFP protein used in our ChIP-seq experiments. Moreover, the sequences bound most avidly by the TF may not necessarily be the most advantageous cis-regulatory sequences to modulate gene expression ${ }^{34,35}$.

Cysteine substitutions in the GATA zinc-finger domain of GtaC did not affect the ability of the protein to translocate to the nucleus but it seemed to completely inactivate its developmental function $^{18}$. Moreover, while the mutations did not completely abolish its ability to bind DNA, they appear to have inhibited direct interaction with GATA-like sequences. GATA zinc-fingers dimerize and can mediate interactions with both DNA and proteins $^{25,36}$ and thus, at least some of the GtaC-DNA interactions could be mediated through other proteins. In addition to cooperative binding, DNA motif preferences can vary depending on the conformational state of TFs when they bind $\mathrm{DNA}^{37}$. Another possibility is the existence of additional, uncharacterized DNA-binding domains in $\mathrm{GtaC}$, which remain unaltered in $\mathrm{GtaC}^{\mathrm{C}-\mathrm{S}}$. While it is tempting to speculate that GtaC binds DNA in different modes, more careful analyses are necessary to characterize the existence and functional consequence of GtaC-DNA interactions. However, regardless of the molecular mechanism, $\mathrm{GtaC}^{\mathrm{C}-\mathrm{S}}$ binding appears to have almost no effect on development because ectopic expression of $\mathrm{GtaC}^{\mathrm{C}-\mathrm{S}}$ in the gtaC-null strain does not significantly modify the developmental or the transcriptional phenotypes.

We integrated DNA-binding and transcriptome data from the $\mathrm{CM}$ and $\mathrm{KO}$ strains to identify putative direct targets of $\mathrm{GtaC}$ and found good concordance between the data sets. Despite ectopic expression of GtaC-GFP in CM, remarkable similarities between the CM and WT strains, not merely on the basis of gross morphologies, but also at the more detailed resolution of their 
transcriptional phenotypes suggest that the artificially constructed $\mathrm{CM}$ strain is a faithful representation of native GtaC-regulatory function. Temporal patterns of $\mathrm{GtaC}$ binding in promoters and expression patterns of the associated genes are largely cotemporaneous, suggesting a causative relationship between TF binding and transcription of targets. Among the $\mathrm{GtaC}$ targets, we found enrichment in two broad developmental processes during the early and late times. Our results are consistent with GtaC's role in cAMP-mediated processes during early development ${ }^{18}$. The enrichment of processes relating to cell differentiation during the late time point broadly agrees with the previously described roles of GtaC in cell-type specification ${ }^{19}$. The temporal regulation of GtaC-DNA binding is consistent with this dual role in development. Since our experimental design did not directly test cell-type-specific functions of $\mathrm{GtaC}$, the possibility that the identified late function may be because of cumulative effects of its early roles, however slim, cannot be completely ruled out.

Another interesting observation is the over-representation of co-regulated orthologues between $D$. discoideum and D. purpureum among $\mathrm{GtaC}$ targets. Given the similarities between the two, our data suggest that some of the GtaC-dependent regulatory modules are conserved between these evolutionarily distant species ${ }^{17}$. Many GtaC targets, such as acaA and carA, are essential for $D$. discoideum development, reinforcing the notion that $\mathrm{GtaC}$ is a critical regulator of development. The functional importance of $\mathrm{GtaC}$ targets is also illustrated by the abundance of putative TFs. A number of these TFs, such as GbfA and CudA, also play important roles in Dictyostelium development ${ }^{10,13}$. The critical role that $\mathrm{GtaC}$ has during $D$. discoideum development, the essential functions it regulates and the proposed evolutionary conservation establish $\mathrm{GtaC}$ as a significant node in the transcriptional regulatory network that governs Dictyostelium development.

Many studies that utilize high-throughput technologies, such as ChIP-seq and RNA-seq, provide a snapshot of the cellular population average ${ }^{38,39}$. These data are invaluable to obtain a coarse overview of the molecular events that underlie broad biological processes. However, the dynamics of TF occupancy and its effect on gene regulation often occur with varying timescales and heterogeneity. Therefore, temporal linkage of these data is essential to understand general features of gene networks; however, it is still difficult to resolve with mere static population averages ${ }^{39}$. The dynamics of intracellular processes are often dependent on mechanisms used in processing and responding to time-varying input signals ${ }^{18,39-41}$. Our previous findings detailed an oscillatory nuclear localization behaviour of $\mathrm{GtaC}$ in response to pulsatile cAMP during the early aggregative phase in development ${ }^{18}$. The $c s a A$ gene (contact site $\mathrm{A}$ ), that we identified as a direct $\mathrm{GtaC}$ target, is transcribed periodically, with periods similar to that of cAMP pulses ${ }^{42}$ and coinciding with $\mathrm{GtaC}$ nuclear accumulation, albeit with a slight delay ${ }^{18}$. Further experimentation, in the presence or absence of pulsatile cAMP, at the single cell level and at finer timescales would be required to test whether this is a general phenomenon of GtaC-dependent transcriptional regulation. Our study provides genome-wide molecular snapshots at different stages of development and it serves as a useful hypothesis generating tool to identify and characterize more such TF-target relationships.

\footnotetext{
Methods

Cell culture and development. We maintained each $D$. discoideum strain at $22^{\circ} \mathrm{C}$ in shaking suspension in HL5 medium with the indicated supplements (Supplementary Table 1). To induce synchronized development, we collected mid-log phase cells and washed them twice in potassium phosphate buffer (KK2) or developmental buffer $(\mathrm{DB})^{18,43}$. Then, we deposited $3.3 \times 10^{6} \mathrm{cells} \mathrm{cm}^{-2}$ on nitrocellulose filters that were placed on a buffer-soaked filter paper pad or
}

$5.2 \times 10^{5}$ cells $\mathrm{cm}^{-2}$ on DB agar. All strains we used in this paper were described in ref. 18 .

Chromatin immunoprecipitation. ChIP using Dictyostelium cells was performed as described in ref. 44 with the following modifications. We used developing CM cells at starvation-sensing (S), early aggregation (E) and late aggregation (L) and on CS cells at the two later time points. As a control for the association of GFP with DNA we performed ChIP on a gtaC-null strain ectopically expressing GFP at the corresponding time points (Supplementary Table 1). We harvested cells at each time point, incubated them with $1 \%$ paraformaldehyde in KK2 buffer at room temperature for $5 \mathrm{~min}$ to crosslink and then added $2.5 \mathrm{M}$ glycine solution to a final concentration of $125 \mathrm{mM}$ to terminate the crosslinking reaction. After washing the cells with $\mathrm{KK} 2$, we isolated nuclei by resuspending the cells in a detergent solution (40 mM Tris- $\mathrm{HCl} \mathrm{pH} 7.8,6 \mathrm{mM} \mathrm{MgCl}_{2}, 40 \mathrm{mM} \mathrm{KCl}, 0.1 \mathrm{mM}$ EDTA, $5 \mathrm{mM}$ dithiothreitol, $1.5 \%$ Sucrose and $0.4 \%$ NP40) for $10 \mathrm{~min}$ on ice ${ }^{45}$. We then resuspended the nuclei in ice-cold lysis buffer $(50 \mathrm{mM}$ Tris- $\mathrm{HCl} \mathrm{pH} 8.0,150 \mathrm{mM}$ $\mathrm{NaCl}, 2 \mathrm{mM}$ EDTA, $1 \%$ Triton X-100 and $0.05 \%$ SDS) containing protease inhibitor cocktail (Roche) and $2 \mathrm{mM}$ phenylmethylsulfonyl fluoride, and sheared the chromatin to 100-300 bp fragments by sonication for nine times at 4-s pulses (amplitude: 45\%) using a digital sonicator (S-250D; Branson). After brief centrifugation, we incubated the supernatants for $30 \mathrm{~min}$ on ice and centrifuged again to remove the insoluble fraction at lower temperature. The nuclear extracts were pre-cleared with protein G Dynabeads (Invitrogen), which were previously blocked with yeast tRNA, glycogen and BSA. We then incubated the pre-cleared extracts with blocked protein G Dynabeads with $(2.4 \mu \mathrm{g})$ or without anti-GFP antibodies (clones 7.1 and 13.1 mixture: 11814460001, Roche) on a rotator, overnight at $4{ }^{\circ} \mathrm{C}$. We washed the beads twice with lysis buffer, twice with high-salt buffer (50 mM Tris- $\mathrm{HCl}$ pH 8.0, $500 \mathrm{mM} \mathrm{NaCl}, 2 \mathrm{mM}$ EDTA, 1\% Triton X-100 and $0.1 \%$ SDS), twice with LiCl-wash buffer ( $10 \mathrm{mM}$ Tris- $\mathrm{HCl} \mathrm{pH} 8.0,250 \mathrm{mM} \mathrm{LiCl}$, $1 \mathrm{mM}$ EDTA, $1 \%$ NP40, and $1 \%$ sodium deoxycholate) and twice with TE buffer, followed by elution with elution buffer $(10 \mathrm{mM}$ Tris-HCl $\mathrm{pH} 7.5,1 \mathrm{mM}$ EDTA and $1 \%$ SDS) twice at $65^{\circ} \mathrm{C}$ for $15 \mathrm{~min}$, reverse-crosslinking at $65^{\circ} \mathrm{C}$ overnight and purification of the ChIP DNA. We then processed the ChIP DNA fragments to generate multiplexed libraries according to the preparation of barcoded sequencing libraries for the Illumina's Genome Analyzer platform ${ }^{46}$ except that we amplified the multiplexed libraries using AccuPrime Pfx DNA polymerase (Life Technologies) at the last step, and performed Illumina sequencing (Illumina HiSeq).

ChIP-seq data analysis. The sequencing data were mapped to the Dictyostelium reference genome ${ }^{47}$ as described in ref. 46 . Briefly, we sequenced the libraries (read length $=50$ bases) and the resulting FASTQ files were mapped using the alignment tool bowtie ${ }^{48}$ (version 0.12 .7 ) allowing only for single hits $(-\mathrm{m} 1)$ and trimming unmapped reads up to $10 \mathrm{bp}$ iteratively by $2 \mathrm{bp}$. To identify genomic regions preferentially associated with $\mathrm{GtaC}$, we performed peak calling using custom $\mathrm{R}$ scripts. Briefly, peak calling was performed on $5-\mathrm{kb}$ genomic regions using partially overlapping ( $50 \%$ overlap), sliding windows of $200 \mathrm{bp}$. For each window, we calculated the log ratio of the scaled coverage (scaled by number of mapped reads) between sample and control ChIP-seq experiments while permitting a maximum of five mapped reads to share the same genomic coordinates. At each time point, we identified $\mathrm{GtaC}$ peaks as genomic regions preferentially associated with $\mathrm{GtaC}$ in the sample compared with control (enrichment $>4$-fold; false discovery rates $($ FDR $)<0.01$; read support $>50)$. In the proximity of candidate peaks, we accounted for shifts (up to $200 \mathrm{bp}$ ) in the distributions of reads mapping to positive and negative strands ${ }^{49}$ and enforced similar read support on either strand (absolute $\log$ ratio $<0.5$ ). In addition, we used $\mathrm{MACS}^{50}$ to identify peaks (parameters: shiftsize $=150$, bw (band width $)=200$, slocal ( small local region $)=5,000)$. Only peaks that were commonly identified using both methods were used, and we focused on peaks in intergenic regions for further analyses. We used MEME$\mathrm{ChIP}^{50}$ (motif lengths 4-12 bp; E-value threshold: $10^{-3}$ ) to search for overrepresented motifs on the 600 most enriched GtaC- and $\mathrm{GtaC}^{\mathrm{C}-\mathrm{S}}$ peaks.

To associate peaks with genes, we defined the promoter region of a gene as the region $5^{\prime}$ of the ORF, up to the nearest upstream gene, and assigned all peaks within a promoter region to the gene. We calculated the binding scores of each gene using all peaks in its promoter region on the basis of a scoring scheme adapted from refs 51,52 with an exponentially decreasing function of its percent distance to the gene start identified on the basis of existing gene models obtained from dictyBase (http://dictybase.org/), weighted by the differential enrichment in GtaC coverage between sample and control ChIP-seq experiments.

RNA-seq. We collected RNA samples in duplicates from WT, CM, KO and CS strains (Supplementary Table 1). The time points sampled corresponded to the vegetative growth $(\mathrm{V} ; 0 \mathrm{~h})$, starvation-sensing $(\mathrm{S} ; 1-2 \mathrm{~h})$, early aggregation $(\mathrm{E} ; 3-6 \mathrm{~h})$ and late aggregation $(\mathrm{L} ; 7-9 \mathrm{~h})$ stages of WT development. The data sets from WT, CM and KO strains at starvation-sensing, early aggregation and late aggregation were published in ref. 18, and the raw data were deposited under the accession number GSE54866 in the Gene Expression Omnibus. We prepared multiplexed cDNA libraries using these RNA samples as described ${ }^{18,46}$ and sequenced them on Illumina HiSeq. 
Transcriptome analyses. The resulting sequences were mapped to the Dictyostelium reference genome ${ }^{46}$. Relative distances between the transcriptomes were visualized using classical MDS (R function cmdscale) and using hierarchical clustering with bootstrapping ( $\mathrm{R}$ package 'pvclust' version $1.2-2)^{53}$ with optimized leaf ordering (R package 'cba' version $0.2-14$ ). We used SC to calculate the distance $(D=1-\mathrm{SC})$ and complete linkage as the clustering criterion. The transcriptome analyses yielded data on the steady-state mRNA levels of 12,435 genes, $\sim 3 \%$ of which were not expressed under any of the conditions sampled (Supplementary Data 1). In all cases, the biological replicates were very similar to one another $(\mathrm{SC} \geq 0.97)$.

We performed differential expression (DE) analyses using baySeq ${ }^{54}$ as described in ref. 55. Briefly, we compared the transcriptomes of each genotype and developmental time point to one another and used custom R scripts (baySeq R package version 1.16.0) to perform these analyses. For each comparison, we considered genes with FDR lower than 0.05 and likelihoods greater than 0.9 to be differentially expressed. We identified genes that were developmentally regulated over time in WT and CM cells and differentially expressed between WT and KO, $\mathrm{CM}$ and $\mathrm{KO}$, WT and CS, and CM and CS at any time point. Their standardized mRNA abundances were visualized as a heatmap ( $\mathrm{R}$ function heatmap.2). At each time point we categorized differentially expressed genes as UP or DOWN if their average normalized mRNA abundance was higher or lower, respectively, in CM compared with KO cells. Genes that were differentially expressed between $\mathrm{KO}$ and CS cells were also categorized as UP or DOWN if their average normalized mRNA abundances were higher or lower, respectively, in CS compared with KO cells. In $\mathrm{DE}$ comparisons between two genotypes, we calculated the DE scores of genes with FDR $<0.05$ as the log ratio of their average normalized expression on the two genotypes. For all other genes, DE scores for this comparison were set to 0 .

Combining transcriptome and binding data. We identified the number of genes that were bound by $\mathrm{GtaC}$ and $\mathrm{GtaC}^{\mathrm{C}-\mathrm{S}}$ and differentially expressed between $\mathrm{CM}$ and $\mathrm{KO}$ and $\mathrm{CS}$ and $\mathrm{KO}$ strains, respectively, at both $\mathrm{E}$ and $\mathrm{L}$, and visualized the overlap as Venn diagrams ( $\mathrm{R}$ package 'Vennerable' version 3.0/r82, R function Venn). To test whether the proportion of overlapping genes identified in our data was different from expected by chance, we randomly sampled the same number of genes from the genome, as observed in our data, at both time points. We counted the number of overlapping genes and obtained an empirical distribution by repeating this procedure a million times. At each time point, we used this distribution to calculate the number of instances that showed a larger overlap than in our data and found that probability of such an event was close to zero. In order to test for regulatory relationships between TF binding in the promoters and mRNA abundance of the downstream genes, we obtained distributions ( $\mathrm{R}$ package 'Hmisc' version 3.14-4, R function Ecdf) of $\mathrm{GtaC}$ - and $\mathrm{GtaC}^{\mathrm{C}-\mathrm{S}}$-binding scores on genes categorized as UP, DOWN or NDE at each time point in CM and CS cells. We used a nonparametric statistical test (Kolmogorov-Smirnov test; R function ks. test) to compare the binding score distributions of genes that are differentially expressed (UP or DOWN) and those that are not (NDE).

To identify enriched short DNA sequences among developmental-stage-specific gene sets, we first identified genes showing large changes in gene expression in $\mathrm{CM}$ compared with $\mathrm{KO}$ cells (absolute log-ratio $>2$ ) at each of the three time points. Then, we used MEME-ChIP ${ }^{56}$ on the promoter regions of the 600 most enriched GtaC-bound peaks to identify enriched motifs.

We ranked the genes on the basis of the absolute values of their $\mathrm{DE}$ scores in comparisons between CM and KO strains (genes with highest values first) at each time point. We also ranked genes on the basis of their GtaC-binding scores such that genes with the high binding scores were ranked first. We computed the RP of genes on the basis of their ranked DE scores and their ranked binding scores at each time point ${ }^{7}$ and genes with lower RPs $(\mathrm{GtaC}-\mathrm{RP}<0.005)$ were considered to be direct targets of GtaC. We performed Gene Ontology enrichment analyses using custom R scripts (R package 'topGO' version 2.14.0). The GO annotation files for D. discoideum were obtained from dictyBase (http://dictybase.org/).

\section{References}

1. Peter, I. S. \& Davidson, E. H. Evolution of gene regulatory networks controlling body plan development. Cell 144, 970-985 (2011).

2. Spitz, F. \& Furlong, E. E. M. Transcription factors: from enhancer binding to developmental control. Nat. Rev. Genet. 13, 613-626 (2012).

3. Li, G. et al. Temporal patterns of gene expression in developing maize endosperm identified through transcriptome sequencing. Proc. Natl Acad. Sci. USA 111, 7582-7587 (2014).

4. Thompson, C. L. et al. A high-resolution spatiotemporal atlas of gene expression of the developing mouse brain. Neuron 83, 309-323 (2014)

5. Grün, D. et al. Conservation of mRNA and protein expression during development of C. elegans. Cell Rep. 6, 565-577 (2014).

6. Park, P. J. ChIP-seq: advantages and challenges of a maturing technology. Nat. Rev. Genet. 10, 669-680 (2009).

7. Wang, S. et al. Target analysis by integration of transcriptome and ChIP-seq data with BETA. Nat. Protoc. 8, 2502-2515 (2013).

8. Parikh, A. et al. Conserved developmental transcriptomes in evolutionarily divergent species. Genome Biol. 11, R35 (2010).
9. Rosengarten, R. D., Santhanam, B. \& Katoh-Kurasawa, M. in Dictyostelids: Evolution, Genomics and Cell Biology. (eds Romeralo, M., Baldauf, S. \& Escalante, R.) 89-108 (Springer, 2013).

10. Hjorth, A. L., Pears, C., Williams, J. G. \& Firtel, R. A. A developmentally regulated trans-acting factor recognizes dissimilar G/C-rich elements controlling a class of cAMP-inducible Dictyostelium genes. Genes Dev. 4, 419-432 (1990).

11. Kawata, T. et al. SH2 signaling in a lower eukaryote: a STAT protein that regulates stalk cell differentiation in Dictyostelium. Cell 89, 909-916 (1997).

12. Escalante, R. \& Sastre, L. A Serum Response Factor homolog is required for spore differentiation in Dictyostelium. Dev. Camb. Engl. 125, 3801-3808 (1998).

13. Fukuzawa, M., Hopper, N. \& Williams, J. cudA: a Dictyostelium gene with pleiotropic effects on cellular differentiation and slug behaviour. Dev. Camb. Engl. 124, 2719-2728 (1997).

14. Kessin, R. H. Dictyostelium: Evolution, Cell Biology, and the Development of Multicellularity (Cambridge University Press, 2001).

15. Williams, J. G. Transcriptional regulation of Dictyostelium pattern formation. EMBO Rep. 7, 694-698 (2006).

16. Schaap, P. et al. Molecular phylogeny and evolution of morphology in the social amoebas. Science 314, 661-663 (2006).

17. Sucgang, R. et al. Comparative genomics of the social amoebae Dictyostelium discoideum and Dictyostelium purpureum. Genome Biol. 12, R20 (2011).

18. Cai, H. et al. Nucleocytoplasmic shuttling of a GATA transcription factor functions as a development timer. Science 343, 1249531 (2014).

19. Keller, T. \& Thompson, C. R. L. Cell type specificity of a diffusible inducer is determined by a GATA family transcription factor. Dev. Camb. Engl. 135, 1635-1645 (2008).

20. Kessin, R. H. Two different genomes that produce the same result. Genome Biol. 11, 114 (2010).

21. Loomis, W. F. \& Shaulsky, G. Developmental changes in transcriptional profiles. Dev. Growth Differ. 53, 567-575 (2011).

22. Galardi-Castilla, M., Garciandía, A., Suarez, T. \& Sastre, L. The Dictyostelium discoideum acaA gene is transcribed from alternative promoters during aggregation and multicellular development. PLoS ONE 5, e13286 (2010).

23. Louis, J. M., Saxe, C. L. \& Kimmel, A. R. Two transmembrane signaling mechanisms control expression of the cAMP receptor gene CAR1 during Dictyostelium development. Proc. Natl Acad. Sci. USA 90, 5969-5973 (1993).

24. Omichinski, J. G. et al. NMR structure of a specific DNA complex of Zn-containing DNA binding domain of GATA-1. Science 261, 438-446 (1993).

25. Matthews, J. M. \& Sunde, M. Zinc fingers--folds for many occasions. IUBMB Life 54, 351-355 (2002).

26. Ringnér, M. What is principal component analysis? Nat. Biotechnol. 26, 303-304 (2008).

27. Iranfar, N., Fuller, D. \& Loomis, W. F. Genome-wide expression analyses of gene regulation during early development of Dictyostelium discoideum. Eukaryot. Cell 2, 664-670 (2003).

28. Jakobsen, J. S. et al. Temporal ChIP-on-chip reveals Biniou as a universal regulator of the visceral muscle transcriptional network. Genes Dev. 21, 2448-2460 (2007).

29. Jakobsen, J. S. et al. Temporal mapping of CEBPA and CEBPB binding during liver regeneration reveals dynamic occupancy and specific regulatory codes for homeostatic and cell cycle gene batteries. Genome Res. 23, 592-603 (2013).

30. Pires-daSilva, A. \& Sommer, R. J. The evolution of signalling pathways in animal development. Nat. Rev. Genet. 4, 39-49 (2003).

31. Loomis, W. F. Cell signaling during development of Dictyostelium. Dev. Biol. 391, 1-16 (2014).

32. Purvis, J. E. \& Lahav, G. Encoding and decoding cellular information through signaling dynamics. Cell 152, 945-956 (2013).

33. Weirauch, M. T. et al. Determination and inference of eukaryotic transcription factor sequence specificity. Cell 158, 1431-1443 (2014).

34. Jiang, J. \& Levine, M. Binding affinities and cooperative interactions with bHLH activators delimit threshold responses to the dorsal gradient morphogen. Cell 72, 741-752 (1993).

35. Dadiani, M. et al. Two DNA-encoded strategies for increasing expression with opposing effects on promoter dynamics and transcriptional noise. Genome Res. 23, 966-976 (2013).

36. Gronenborn, A. M. Zinc Finger Proteins: From Atomic Contact to Cellular Function (Landes Bioscience; Kluwer Academic/Plenum Publishers, 2005).

37. Siggers, T. \& Gordân, R. Protein-DNA binding: complexities and multi-protein codes. Nucleic Acids Res. 42, 2099-2111 (2014).

38. Sanchez, A. \& Golding, I. Genetic determinants and cellular constraints in noisy gene expression. Science 342, 1188-1193 (2013).

39. Voss, T. C. \& Hager, G. L. Dynamic regulation of transcriptional states by chromatin and transcription factors. Nat. Rev. Genet. 15, 69-81 (2014).

40. Hao, N. \& O'Shea, E. K. Signal-dependent dynamics of transcription factor translocation controls gene expression. Nat. Struct. Mol. Biol. 19, 31-39 (2012). 
41. Wollman, R. Development. Counting the ways to decode dynamic signals. Science 343, 1326-1327 (2014).

42. Corrigan, A. M. \& Chubb, J. R. Regulation of transcriptional bursting by a naturally oscillating signal. Curr. Biol. 24, 205-211 (2014).

43. Huang, E. et al. BzpF is a CREB-like transcription factor that regulates spore maturation and stability in Dictyostelium. Dev. Biol. 358, 137-146 (2011).

44. Dubin, M., Fuchs, J., Gräf, R., Schubert, I. \& Nellen, W. Dynamics of a novel centromeric histone variant $\mathrm{CenH} 3$ reveals the evolutionary ancestral timing of centromere biogenesis. Nucleic Acids Res. 38, 7526-7537 (2010).

45. Katoh, M. et al. An orderly retreat: dedifferentiation is a regulated process. Proc. Natl Acad. Sci. USA 101, 7005-7010 (2004).

46. Miranda, E. R. et al. Transcriptional profiling of Dictyostelium with RNA sequencing. Methods Mol. Biol. 983, 139-171 (2013).

47. Eichinger, L. et al. The genome of the social amoeba Dictyostelium discoideum. Nature 435, 43-57 (2005)

48. Langmead, B., Trapnell, C., Pop, M. \& Salzberg, S. L. Ultrafast and memoryefficient alignment of short DNA sequences to the human genome. Genome Biol. 10, R25 (2009)

49. Jothi, R., Cuddapah, S., Barski, A., Cui, K. \& Zhao, K. Genome-wide identification of in vivo protein-DNA binding sites from ChIP-Seq data. Nucleic Acids Res. 36, 5221-5231 (2008)

50. Zhang, Y. et al. Model-based analysis of ChIP-Seq (MACS). Genome Biol. 9, R137 (2008).

51. Ouyang, Z., Zhou, Q. \& Wong, W. H. ChIP-Seq of transcription factors predicts absolute and differential gene expression in embryonic stem cells. Proc. Natl Acad. Sci. USA 106, 21521-21526 (2009).

52. Sikora-Wohlfeld, W., Ackermann, M., Christodoulou, E. G., Singaravelu, K. \& Beyer, A. Assessing computational methods for transcription factor target gene identification based on ChIP-seq data. PLoS Comput. Biol. 9, e1003342 (2013).

53. Suzuki, R. \& Shimodaira, H. pvclust: Hierarchical Clustering with P-Values via Multiscale Bootstrap. $R$ package version 1.2-2, http://cran.r-project.org/web/ packages/pvclust/index.html (2011).

54. Hardcastle, T. J. \& Kelly, K. A. baySeq: empirical Bayesian methods for identifying differential expression in sequence count data. BMC Bioinformatics 11, 422 (2010).

55. Nasser, W. et al. Bacterial discrimination by dictyostelid amoebae reveals the complexity of ancient interspecies interactions. Curr. Biol. 23, 862-872 (2013).

56. Machanick, P. \& Bailey, T. L. MEME-ChIP: motif analysis of large DNA datasets. Bioinformatics 27, 1696-1697 (2011)

57. Bailey, T. L. et al. MEME SUITE: tools for motif discovery and searching. Nucleic Acids Res. 37, W202-W208 (2009).
58. Bailey, T. L. DREME: motif discovery in transcription factor ChIP-seq data. Bioinformatics 27, 1653-1659 (2011).

\section{Acknowledgements}

We thank Gregor Rot, Marko Toplak and Blaž Zupan from the University of Ljubljana for assistance with data management and analyses. We also thank Zhangdong Liu at the Department of Pediatrics, Baylor College of Medicine and Marinka Žitnik and Blaž Zupan at the University of Ljubljana for useful suggestions and helpful discussions. This work was supported by the National institutes of Health 'the Dictyostelium Functional Genomics Program Project Grant' (P01 HD39691) to G.S., R01 GM28007 and R01 GM34933 to P.N.D. and Helen Hay Whitney postdoctoral fellowship to H.C.

\section{Author contributions}

M.K.-K., B.S. and G.S. conceived and designed the experiments. M.K.-K., B.S. and H.C performed the experiments, and B.S. and M.K.-K. analysed data. P.N.D. and H.C. generated the strains. B.S., M.K.-K. and G.S. wrote the initial manuscript and all authors contributed to the discussion. M.K.-K. and G.S. supervised the study.

\section{Additional information}

Accession codes: The relevant data sets have been deposited in the Gene Expression Omnibus database of National Center for Biotechnology Information under the accession number GSE63151.

Supplementary Information accompanies this paper at http://www.nature.com/ naturecommunications

Competing financial interests: The authors declare no competing financial interests

Reprints and permission information is available online at http://npg.nature.com/ reprintsandpermissions/

How to cite this article: Santhanam, B. et al. The GATA transcription factor GtaC regulates early developmental gene expression dynamics in Dictyostelium. Nat. Commun. 6:7551 doi: 10.1038/ncomms8551 (2015).

(c) (i) This work is licensed under a Creative Commons Attribution 4.0 International License. The images or other third party material in this article are included in the article's Creative Commons license, unless indicated otherwise in the credit line; if the material is not included under the Creative Commons license, users will need to obtain permission from the license holder to reproduce the material. To view a copy of this license, visit http://creativecommons.org/licenses/by/4.0/ 